\title{
Desde los Estados Unidos y Rusia: una mirada a la R2P1
}

\section{From the United States and Russia: a look at the R2P}

\section{Ximena Cujabante (iD}

Universidad Militar Nueva Granada

ximena.cujabante@unimilitar.edu.co

\section{Sara P. Quintero Cordero (iD}

Universidad Militar Nueva Granada

sara.quintero@unimilitar.edu.co

\section{Resumen}

Gracias a los avances teóricos y a la apertura de las Relaciones Internacionales a diferentes enfoques sociales y lingüísticos, se ha logrado ampliar el espectro al momento de identificar problemáticas y asuntos de una investigación. Una de estas ventajas es poder analizar los discursos de diferentes dirigentes políticos e instituciones estatales para vislumbrar su entramado de conceptos, significados e ideas referentes a un tema. En este trabajo lo que se pretende es, por medio del análisis del discurso, entender la noción de seguridad que evocan Estados Unidos y Rusia, para así poder tener mayor información a la hora de analizar las acciones que responden en nombre de la "Responsabilidad de proteger".

Palabras clave: Responsabilidad de proteger, seguridad, construcción.

\begin{abstract}
Thanks to the theoretical advances and the opening of International Relations to different social and linguistic approaches, it has been possible to broaden the spectrum when identifying problems and issues of a research, one of these advantages is to be able to analyze the speeches of different political leaders and state institutions to be able to glimpse its web of concepts, meanings and ideas related to a topic. In this work, what is intended is, through discourse analysis, to understand the way in which the security evoked by the United States and Russia is understood, in order to have more information when analyzing the actions that respond on behalf of the "Responsibility to protect".
\end{abstract}

Keywords: Responsibility to protect, security, construction.

Articulo: Recibido el 02 de febrero de 2019 y aprobado 18 de octubre de 2019.

\section{Cómo citar este artículo:}

Cujabante, X. \& Quintero, S. (2019). Desde los Estados Unidos y Rusia: una mirada a la R2P. Reflexión Politica 21(43), pp. 9-21, doi: 10.29375/01240781.3503

${ }^{1}$ Este artículo es producto de investigación financiado por la Universidad Militar Nueva Granada, titulado: "Responsabilidad de proteger: ¿un factor de inestabilidad a la seguridad internacional o un llamado ético internacional?" INV - EES - 2599. 


\section{Introducción}

"Todo hombre, con tal que no viole las leyes de la justicia, debe quedar en libertad de ejercer su propio interés"

Adam Smith

En los últimos periodos presidenciales, Estados Unidos afronta y desarrolla de manera constante estrategias para la implementación de las relaciones exteriores con el objetivo de mejorar social y económicamente al país, construyendo nuevas pautas para la responsabilidad de proteger de forma dinámica y confiable para el entorno internacional, especialmente con Rusia. Sin lugar a dudas, la globalización y los cambios constantes en otras políticas exteriores a nivel mundial, dificultan la evolución constante de la política en transición actual que lleva Estados Unidos, país que a través de los años ha procurado tener una relación diplomática con Rusia. Sin embargo, en los recientes años esta relación se ha deteriorado por varios factores, entre los cuales está la ideología política y desacuerdo entre los dos últimos presidentes estadounidenses (B. Obama y D. Trump) con el gobierno ruso. A tal punto que en la presidencia de Trump, ocasionó un desplome diplomático internacional grave que afectó considerablemente diversos sectores de la economía para los dos países. Por ende,

El conjunto de políticas, decisiones y acciones que integran un cuerpo de doctrina coherente y consistente, basados en principios claros, sólidos e inmutables, forjados a través de su evolución y experiencia histórica, permanentemente enriquecido y mejorado por el que cada Estado $u$ otro actor o sujeto de la sociedad internacional define su conducta y establece metas y cursos de acción en todos los campos y cuestiones que trasciendan sus fronteras o que puedan repercutir al interior de los suyos, ya que es aplicado sistemáticamente con el objeto de encausar y aprovechar el entorno internacional para el mejor cumplimiento de los objetivos trazados en aras del bien general de la nación, así como de la búsqueda del mantenimiento de las relaciones armoniosas con el exterior (Hernández, 2006, p. 152).

La responsabilidad de proteger es prolongación de una estrategia interna, y refleja más directamente el interés de un país, o de sectores hegemónicos del mismo, bien sea de manera conjunta o fragmentada. Es decir, no existe el interés nacional sino una sumatoria de intereses en interacción permanente. Por ejemplo, en Estados Unidos manejan la acumulación de capital para bienestar propio, es decir, para gastos internos como armamento y apoyo a posibles guerras.

El enfoque presentado previamente brinda matices cualitativos a la investigación, en la medida en que aporta herramientas para explicar las razones por las cuales ciertos Estados y mandatarios actúan de una forma y no de otra. De igual forma, también permite dar seguimiento a la manera como se entiende la intervención y seguridad humanitaria y, en específico, cómo se da el uso del concepto de responsabilidad de proteger. Este aspecto se identificará por medio del análisis del discurso de los mandatarios y delegados de Estados Unidos en el caso de Libia para el año 2011, y Rusia en el 2008 con Georgia, para poder analizar las percepciones que se tienen del concepto y cómo actúan según sus intereses.

La elección de los casos de estudio de esta investigación es con el fin último de entender las posiciones orientadoras frente a un tema controversial, como lo es el caso de la justificación del uso o no de la responsabilidad de proteger por parte de grandes Estados, como Estados Unidos y Rusia. Estados que tienen posturas divergentes que se ven permeadas gracias a su ubicación geoestratégica, poderío económico, militar y político. La primera parte de este artículo se enfocará en la utilización de la R2P desde lo discursivo por el expresidente Barack Obama, para entender cómo este concepto es utilizado desde el punto de vista político-militar. En la segunda parte, se centrará el análisis sobre el papel que desempeña Rusia dentro del uso de la responsabilidad de proteger, para entender de qué manera se han construido 
los intereses a partir de la interacción que ha tenido este Estado en el sistema internacional.

La metodología que se utilizará es cualitativa y la herramienta que se empleará es el análisis del discurso de altos mandatarios y delegados ante la ONU por parte de Estados Unidos en el 2011, y en el 2008 para el caso de Rusia. El propósito es entender y comparar las percepciones y construcciones de identidad a nivel internacional ante los demás Estados y sociedad, que se generan a partir del uso del término de la responsabilidad de proteger. A partir de lo anterior, se espera brindar aportes al entendimiento de las acciones de dichos Estados.

\section{Estados Unidos vs. Rusia}

\author{
"Si pensamos que algo \\ va mal, debemos actuar \\ en congruencia con ese \\ conocimiento".
}

Tony Judt

La sociedad ha tenido muchos cambios en el último siglo debido a que se han presentado los acontecimientos más importantes de la historia, los cuales han tenido diferentes consecuencias, buenas y malas, en sus sitios de origen y por supuesto a nivel mundial. En esta época se encuentran acontecimientos importantes como la globalización, y su afectación actual para ésta. Es así como la sociedad está en constante cambio, y algunos de ellos han permitido que surjan inseguridades físicas que involucran la persona como tal, y económicas que afectan desde el Estado hasta el individuo.

Sin embargo, la "responsabilidad de proteger" pareciera que surge de malas políticas y decisiones gubernamentales actuales de Estados Unidos y Rusia por afectar estrategias de bienestar común, alejándose de favorecer a la sociedad y a la libertad misma, que permite que los individuos actúen apoyando al Estado de manera democrática y voluntaria.

Los problemas sociales provienen de manejos inadecuados de las riquezas de Estados Unidos y Rusia, allí los bienes en la actualidad son para aquellos que tienen más poder y las masas minoritarias poseen problemas económicos graves, por lo cual se desencadena una desigualdad social. Es el ejemplo de Estados Unidos, donde se presenta esta situación; sin embargo, no cambian su estilo de Estado, dado que no lo ven conveniente por las políticas nuevas que se deberían aplicar, incluyendo la "responsabilidad de proteger" que involucra de manera directa a la ciudadanía. Revisando un poco la historia, Tony Judt expresa que a consecuencia de las guerras mundiales, países como Estados Unidos y algunos de Europa se dieron cuenta de que dichos acontecimientos provocaron

[...] Con las tensiones derivadas de la globalización y las crisis que la acompañan, el Estado tendrá que intervenir cada vez más para resolver conflictos. $\mathrm{Al}$ ser la única institución que se encuentra entre los individuos y los actores no estatales, como los bancos y las corporaciones internacionales, la única instancia reguladora que ocupa el espacio entre los órganos transnacionales y los intereses locales, es probable que el Estado territorial acreciente su importancia política (Judt, 2010, p.509).

Es así como, luego de analizar esta problemática, los países más desarrollados y sobre todo Estados Unidos y Europa, implantaron soluciones que funcionaron a la perfección durante cuatro décadas, donde mejoró sustancialmente el bienestar de estos países. Se hicieron mejoras en el transporte, la cultura, se implementaron diferentes subsidios, y de manera general el bienestar ciudadano, que en teoría respaldaban el compromiso político ante las Naciones Unidas denominados seguridad humana y objetivos del milenio. Sin embargo, como es de saber y se ha comprobado a través de la historia, esta situación de estabilidad y bienestar no duró mucho, después del acontecimiento primordial de los años setenta, pero esto se ha mantenido hasta la actualidad.

El conflicto diplomático permite que los países crezcan, sin embargo esto no significa que puedan tener igualdad y prosperidad las relaciones interestatales entre Estados potencias. Lo cual lleva a concluir que la globalización, así como los problemas diplomáticos, e interdependencia, es un fenómeno que puede afectar y vulnerar a una sociedad de manera positiva o negativa y Estados Unidos no es la excepción.

Es importante mencionar que las alteraciones de conflicto que ha tenido Estados Unidos con Rusia, están impregnadas de la economía y el poder que manejan al interior de sus Estados. Por ello, "Se pensaba que el mercado no era lo más adecuado para definir los objetivos 
colectivos: el Estado tendría que intervenir y llenar el vacío." (Judt, 2010, p. 161). Y esto ha sucedido en las dos grandes potencias a nivel mundial. Se puede afirmar que a futuro los líderes de los Estados mencionados y el mercado libre que conlleva una posible evolución no sean la solución correcta para las problemáticas actuales, por ello se plantea un Estado fuerte y autoritario. La sociedad lo aceptaría. Dada la pérdida que ha tenido la figura social democrática que surgió después de la Segunda Guerra Mundial, ya no posee atractivos para convencer a los ciudadanos de nuevas políticas y terminan perdiendo la apuesta, así como lo ha hecho el conservatismo, que ha perdido grandes principios tales como la moderación social (Judt, 2010).

\section{Identificación o construcción de intereses entre Estados Unidos y Rusia}

Los Estados actúan de forma racional en la medida en que se sopesen los beneficios y los costos y a partir de esto, se determina si es un interés nacional o no. Por otra parte, si se analiza desde la perspectiva constructivista, se evidencia claramente que la forma como se construyen los intereses responde a una lógica donde se tienen en cuenta las percepciones del otro, es decir, a partir de la otredad, cómo se concibe, qué conocimiento previo hay, entre otros. Es importante tener en cuenta el debate sobre agente-estructura, ya que el rompimiento de esto se da en la forma en la que se analiza que las relaciones entre agentes y estructura puede evolucionar y mutar, en la medida en que los agentes interactúan, forman relaciones sociales constitutivas, es decir, "Una relación constitutiva establece una conexión conceptualmente necesaria o lógica entre X y Y, en contraste a la conexión contingente entre entidades existentes independientemente de las relaciones causales" (Wendt, 1995, p. 72). Se demuestra así que en la medida en que existan percepciones de amenaza entre Estados, surgirán relaciones e identidades de cooperación u hostilidad, lo cual también explica las razones por las cuales existen coaliciones o alianzas entre Estados en el sistema internacional.

Dentro de las relaciones internacionales, la soberanía internacional y la anarquía en el sistema internacional entre Estados Unidos y Rusia son piedra angular en los estudios clásicos. Si partimos de esto, variedad de autores realistas resaltan el rol de los factores materiales, las capacidades militares, las amenazas y la defensa en la seguridad estatal, como bien lo subrayan algunas definiciones: "Los realistas consideran que los principales actores en el ámbito internacional son los Estados, que se preocupan por su propia seguridad, actúan en pos de sus propios intereses nacionales y luchan por el poder" (Sánchez, 2006, p. 126).

En este sentido, Estados Unidos y Rusia parten del reconocimiento del otro y se adopta una postura que determina cuál es el rol y el tipo de relación que se entabla, si es visto como aliado o enemigo, es decir, que se empieza a construir bajo la idea de una comparación. Esto último es entendido desde el ámbito sociológico y antropológico como la otredad. En el campo de las relaciones internacionales, David Campbell lo aterriza de la siguiente manera "asegurar un yo ordenado y un mundo ordenado -particularmente cuando el campo sobre el que se presenta este proceso es tan costoso como un Estado- implica definir elementos que se ponen en el camino del orden como formas de alteridad" (Campbell, 1992, p. 55). Todas las circunstancias de conflicto entre las dos potencias, se basan en la "Responsabilidad de Proteger" , y a su manera le dan la interpretación en el derecho internacional.

Ejemplo de esto se puede ver en el reconocimiento internacional del Estado de Palestina. En la medida en que todos los Estados lo reconozcan como un actor independiente y autónomo en las relaciones internacionales, el Estado de Palestina existe y por ende, se establecen relaciones sociales, comerciales y políticas con este. Pero si, por el contrario, no se le reconoce como tal, no hay interacciones, es como si no existiese; en la medida en que no haya reconocimiento se niega el sujeto, no existe. Estos elementos son referidos en el constructivismo por medio de la intersubjetividad, en donde por medio no solo del reconocimiento del otro, sino también gracias a la creación de significados junto con el otro es que se construyen las relaciones, y para Schutz esta construcción se genera a partir de la cotidianidad (Romero y Sosa, 2007). Lo anterior lo expone uno de los autores más influyentes en el constructivismo, Alexander Wendt:

La distribución de poder siempre puede afectar los cálculos de los Estados, pero el cómo lo hace depende de la intersubjetividad, entendimientos y expectativas, en la "distribución de conocimiento", 
que constituye sus concepciones de sí mismo y del otro. [...] Son los significados colectivos los que constituyen las estructuras que organizan nuestras acciones (Wendt, 1992, p. 397).

$\mathrm{Si}$ se parte del entendimiento de que las teorías y conceptos evolucionan a lo largo del tiempo se podrán identificar estos cambios en el ámbito de las relaciones internacionales, iniciando con concepciones completamente centradas en la seguridad, el rol principal del Estado y la soberanía. Estas han mutado y concebido pertinentes las relaciones económicas, interdependencia, terrorismo, rol del narcotráfico, llegando así a concebir otros sujetos que afectan las interacciones entre Estados y al mismo sistema internacional. Es gracias a aquellos sujetos silenciados, como por ejemplo las mujeres, los individuos, las fuerzas del mercado, el medio ambiente, entre otros, que se logra irrumpir desde otras esferas al estudio del sistema internacional.

Las identidades, los movimientos sociales y las motivaciones de los actores empiezan a estudiarse por medio del análisis del lenguaje, herramienta utilizada para evidenciar la importancia de lograr entender el mundo de las ideas, de la construcción de referentes de poder y la manera en que se mantienen por medio de la legitimidad en las relaciones globales. Como se mencionaba anteriormente, las relaciones entre los sujetos internacionales se basan en las percepciones y en el conocimiento previo que tiene el uno del otro.

Enfatiza la maleabilidad de la identidad colectiva y de la variabilidad de las restricciones de la anarquía como consecuencia. Los Estados no son unidades, cada uno dotado con identidades egoístas por virtud de la estructura del sistema internacional. No es la restricción estructural de la anarquía siempre y en todos lados necesariamente la misma. Una estructura cooperativa se puede desarrollar para reemplazar una de autoayuda (McSweeney, 1999, p. 122).

Donde la transformación en el sistema internacional de las percepciones que hay entre Estados Unidos y Rusia resulta bastante interesante. Dentro de las teorías críticas de las relaciones internacionales surgen diversos marcos de referencia y enfoques, los cuales buscan ser multidisciplinares y lograr el acercamiento a los objetos de estudio desde métodos diferenciados a los tradicionales. Estas buscan perspectivas que brinden enfoques holísticos y mucho más complejos y completos para un mejor entendimiento de las realidades actuales. Dentro de estas teorías, encontramos el constructivismo y el análisis del discurso, las cuales se ven enmarcadas en un entendimiento donde:

Las estructuras fundamentales de las políticas internacionales son sociales más que estrictamente materiales (un reclamo que se opone al materialismo), y que estas estructuras moldean los intereses e identidades de los actores, no solo su comportamiento (un reclamo que se opone al racionalismo) (Wendt, 2005, p. 13).

Por un lado, las relaciones diplomáticas entre Estados Unidos y Rusia son vistas como una ruptura a las tradiciones teóricas e inclusive conceptuales, puesto que estas últimas establecen marcos de referencia que aplican según métodos cuantitativos a la realidad para comprobar su factibilidad; a diferencia del constructivismo el cual es, "en definitiva, una propuesta en la que se concibe el conocimiento como algo provisional, que contempla múltiples construcciones y se forma a través de las negociaciones dentro de los límites de una comunidad" (Pérez, 2005, p. 46). Se evidencia así que incluso hasta la teoría y los elementos utilizados en las investigaciones no son entendidos de la misma manera por todos los actores, sino que es una construcción colectiva, social e incluso constitutiva. En el sistema internacional, las relaciones se constituyen por medio de las estructuras sociales definidas “[...] en parte por compartir entendimientos, expectativas, o conocimiento. Estos constituyen a los actores en una situación y en la naturaleza de sus relaciones, si es cooperativa o conflictual" (Wendt, 1995, p. 73).

\section{Responsabilidad de proteger}

El objeto de estudio de esta investigación es el discurso de la responsabilidad de proteger, el cual entra en las lógicas de cooperación en un ambiente hostil y de constante amenaza, por lo cual hace consonancia con lo anteriormente mencionado. Como ya se mencionó, los Estados son los principales actores que tienen el deber moral y político de intervenir. Mejor explicado:

La responsabilidad de proteger representa un compromiso político para poner fin a las peores formas de violencia y persecución. Busca reducir la brecha entre las obligaciones preexistentes de los Estados miembros en virtud del derecho 
internacional humanitario y de derechos humanos y la realidad que enfrentan las poblaciones en riesgo de genocidio, crímenes de guerra, depuración étnica y crímenes de lesa humanidad (United Nations Office on Genocide Prevention and the Responsability to Protect, s.f.).

Es así como aquellos Estados que tomen o no una postura frente a la intervención humanitaria responden al moldeamiento de sus identidades teniendo como referente las interacciones en la esfera internacional en términos morales de lo que está bien o mal. En un principio, Obama alude al rol que ha interpretado Estados Unidos en el escenario global, mencionando que "Durante generaciones, los Estados Unidos de América han desempeñado un papel único como ancla de la seguridad mundial y como defensores de la libertad humana" (Obama, 2011). En este punto, la identidad estatal como garante de seguridad es clara y evoca la importancia que tiene este Estado dentro del escenario general, ya que, como es aceptado e incluso legitimado hasta cierto punto, Estados Unidos ha sido reconocido como la imagen principal de la lucha por la democracia, y uno de los ideales más cercanos de esta última es la libertad.

Siguiendo con el discurso, Obama se refiere al margen de maniobra en el que se encuentra, "Teniendo en cuenta los riesgos y los costos de la acción militar, somos naturalmente reacios a utilizar la fuerza para resolver los muchos desafios del mundo. Pero cuando nuestros intereses y valores están en juego, tenemos la responsabilidad de actuar" (Obama, 2011). Para entrar en contexto con este tema, hay que mencionar el procedimiento para que la responsabilidad de proteger pueda entrar en rigor, y es por medio de la solicitud al Consejo de Seguridad de la ONU. Cabe aclarar que este tipo de intervención, así sea humanitario, se procura evitar a toda costa, siendo el último recurso para poder tomar acción ante crisis interna de otros Estados. Para determinar que sea imprescindible, hay que primero fijar de manera concisa la intención correcta de la intervención; en segundo lugar, hay que cerciorarse de que las demás amenazas y sanciones políticas, económicas y diplomáticas no hayan surtido efecto y así intervenir. Continuando con las palabras de Obama,

Argumentan que hay muchos lugares en el mundo donde civiles inocentes enfrentan violencia brutal a manos de su gobierno, y no se debe esperar que Estados Unidos vigile el mundo, particularmente cuando tenemos tantas necesidades apremiantes aquí en casa. [...] Y dados los costos y riesgos de la intervención, siempre debemos medir sus intereses frente a la necesidad de acción. Pero eso no puede ser un argumento para nunca actuar en nombre de lo que es correcto. En este país en particular, Libia, en este momento particular, nos enfrentamos con la perspectiva de violencia en una escala horrible. Tuvimos una capacidad única para detener esa violencia: un mandato internacional para la acción, una amplia coalición preparada para unirse a nosotros, el apoyo de los países árabes y un pedido de ayuda del propio pueblo libio. También tuvimos la capacidad de detener a las fuerzas de Gadafi sin poner a las tropas estadounidenses en el suelo (Obama, 2011).

En los intereses de Estados Unidos es claro el papel de la democracia y, relacionado con eso, el salvar vidas en medio de la violencia. Es de esta manera que por medio de la intervención se espera un cambio de régimen político, puesto que uno de los intereses primordiales de Estados Unidos es la implementación del modelo democrático y participativo en un escenario contrario como lo es una dictadura de alrededor de 41 años perpetrada por Muamar el Gadafi (Zoubir y Murillo, 2012).

Desde una perspectiva mucho más crítica, se podría decir que la utilización del discurso de responsabilidad de proteger responde a un antifaz para que de esta manera, se continúe imponiendo la paz democrática por la fuerza, ya que no se tiene en cuenta que los contextos son diferentes, lo que da como resultado Estados con menos herramientas para afrontar casos que deban responder al llamado de responsabilidad de proteger. Se puede explicar de la siguiente manera:

Las realidades del mercado global y del lado indiferente de la globalización pueden afectar la capacidad de un Estado débil para implementar plenamente su responsabilidad de proteger, por ejemplo, socavando la capacidad del gobierno, al exacerbar divisiones étnicas, o al reducir la capacidad del gobierno para ejercer control sobre su territorio y para superar cualquier intento de grupos armados de intimidar segmentos de su población mediante la comisión de crímenes RtoP (Luck, 2009, p. 15).

Es por medio del lenguaje que se implantan las relaciones de amigo-enemigo y como lo menciona el expresidente de Estados Unidos, ya se contaba con el apoyo de la Liga Árabe. También se recibía el apoyo de diversos Estados como lo son Francia y Reino Unido, lo que demuestra que ya se especificaba 
quiénes eran considerados aliados y la identificación de un enemigo en común. Si llegásemos a seguir en el aspecto crítico, se podría decir que esas relaciones sociales entre Estados ante dicha circunstancia también permitían demostrar la influencia que posee Estados Unidos no solo en la región sino también en la ONU, puesto que el protocolo que debía seguir el accionar de la responsabilidad de proteger en términos de intervención en el caso de Libia, era esperar la aprobación para luego tomar acción. Pero Estados Unidos, al tener acceso e incidencia en la zona, ya había ordenado la movilización de naves de guerra en el Mediterráneo (Obama, 2011), pasando por encima del deber ser. Por ende:

\begin{abstract}
A veces, el curso de la historia plantea desafíos que amenazan nuestra humanidad común y nuestra seguridad común: responder a los desastres naturales, por ejemplo; o prevenir el genocidio y mantener la paz; garantizar la seguridad regional y mantener el flujo de comercio. Estos pueden no ser solo problemas de Estados Unidos, pero son importantes para nosotros. Son problemas que vale la pena resolver. Y en estas circunstancias, sabemos que los Estados Unidos, como la nación más poderosa del mundo, a menudo serán llamados a ayudar (Obama, 2011).
\end{abstract}

Es de esta manera que, por medio del discurso, se logra reforzar no solo la imagen de Estados Unidos como una gran potencia, sino que de igual forma, se logra entrever la construcción de una identidad de autorreconocimiento y de la legitimación de esta. Dentro de los análisis de la manera como se emplea el lenguaje en este tipo de discursos, Hansen identifica los intereses directos del empleo de este discurso y es que por medio de "Violaciones al derecho internacional, guerras civiles, hambruna, enfermedades, el SIDA, y la pobreza [...] están articulados como 'problemas de seguridad internacional' en donde la comunidad internacional tiene la responsabilidad de contrarrestar" (Hansen, 2007, pp. 34-35).

En pocas palabras, el empleo del discurso de responsabilidad de proteger genera una cadena causal, al mencionar una problemática que atañe a la comunidad internacional y el deber de responder bajo el principio de responsabilidad. Puesto que como lo menciona Luck, "la responsabilidad de proteger tiene un significado tanto operacional como aspiracional, informando las prácticas y políticas actuales por un lado y por el otro confirmando estándares más altos para ser perseguidos en el tiempo" (Luck, 2009, p.20), y es en el aspecto aspiracional que se evidencia en el discurso el factor moral y del deber ser.

Siguiendo esta línea del deber ser, se puede partir del hecho de reconocer ciertos valores que propenden por ser universales porque representan los intereses y el deber ser de los hechos. Dicho de otra manera "[...] la política internacional está enraizada en la ética que tanto Estados como no actores estatales comparten un vocabulario básico moral que influencia las decisiones individuales o colectivas de los estados" (Amstutz, 2013, p. 8). Ya que, en la medida en que exista un grupo de valores y normas que representen los intereses y los ideales de los Estados, se constituirá una base sólida de un régimen o una institución que pueda actuar en nombre de todos los miembros adscritos, en cuanto, como bien lo menciona Wendt:

Esto contrasta con el sistema de seguridad "cooperativo", en el que los estados se identifican positivamente entre ellos y así la seguridad es percibida como una responsabilidad de todos... Además, se reestructurarán los esfuerzos para hacer avanzar los objetivos propios o la "política de poder", según normas compartidas, y no según el nivel de poder relativo (Wendt, 1992, p. 10).

Se muestra así que en la medida en que Estados Unidos y Rusia se identifiquen con los valores y principios de las normas internacionales, mayor será el vínculo que los une a una causa mayor, es decir, a la cooperación de resolución de conflictos que atañen a la población civil. Cuando las reglas y los principios se ven debilitados o un actor rompe con el orden acordado, diversidad de acciones y acusaciones salen a la luz. En el caso del ataque del jefe de Estado de Libia hacia sus ciudadanos, Obama recalca en sus palabras que:

Hemos intervenido para detener una masacre, y trabajaremos con nuestros aliados y socios para mantener la seguridad de los civiles. Negaremos las armas del régimen, cortamos sus suministros de efectivo, asistiremos a la oposición y trabajaremos con otras naciones para acelerar el día en que Gadafi deje el poder. [...] Con el tiempo y el espacio que hemos proporcionado para el pueblo libio, ellos podrán determinar su propio destino, y así es como debería ser (Obama, 2011).

Mostrando así que en esas relaciones no solo hay una variable que es Gadafi, sino todo 
el entramado de personas que están a favor del régimen dictatorial. Se resalta la última oración de este apartado, referente a la capacidad que tiene el pueblo libio al decidir su destino. Claro está que desde el aspecto político, se evidencia la intencionalidad de poner sobre la mesa el cambio de régimen democrático, donde la población es aquella que es libre de decidir su camino.

A pesar de analizar el discurso oficial de Barack Obama y evidenciar los términos morales que determinan los intereses y la identidad de Estados Unidos, es también enriquecedor para la investigación referirse a la percepción que tuvo el sistema internacional por medio de los medios de comunicación. Al realizar la revisión bibliográfica se presentaron dos posturas en contraposición. Una de ellas fue la inminente victoria que tuvo la intervención humanitaria, en donde medios como CNN declararon que "Debe atribuirse a la administración Obama la elaboración de una política exterior sensata y prudente hacia Libia” (Ellison, 2011).

De igual manera, el New York Times incluso propone declarar este modelo de intervención exitoso como la doctrina Obama, con la salvedad de aclarar que no sería sensato replicarlo de la misma manera en otros escenarios: "Obama presentó esos principios el 28 de marzo [del 2011], cuando dio su único gran discurso sobre el conflicto de Libia, en un discurso en la Universidad George Washington que de muchas maneras estableció los principios de la doctrina de Obama" (Cooper y Myers, 2011). Por último, para recalcar este aspecto positivo, la $\mathrm{BBC}$ hace hincapié en el esfuerzo necesario en conjunto de Estados Unidos, Reino Unido y Francia, y cómo a partir del trabajo en conjunto se pudo dar fin al gobierno de Gadafi (BBC, 2011).

Por otra parte, algunas publicaciones académicas desacreditan el rol de Estados Unidos en Libia e incluso ponen en evidencia los daños y efectos colaterales que esta acción causó. Una de ellas es Foreign Affairs, la cual titula el artículo así: Cómo una bien intencionada intervención terminó en fracaso. Allí se da un recuento de todos los efectos que causó a largo plazo la intervención en nombre de responsabilidad de proteger:

En retrospectiva, la intervención de Obama en Libia fue un fracaso abyecto, juzgado incluso por sus propios estándares. Libia no solo no ha logrado evolucionar hacia una democracia; se ha convertido en un estado fallido. Las muertes violentas y otros abusos contra los derechos humanos se han multiplicado por varias. En lugar de ayudar a Estados Unidos a combatir el terrorismo, como lo hizo Gadafi durante su última década en el poder, Libia ahora sirve como un refugio seguro para las milicias afiliadas tanto a $\mathrm{Al}$ Gaeda como al Estado Islámico de Irak y al-Sham (isis) (Kuperman, 2015).

Siguiendo esta misma línea crítica, un artículo en Foreign Policy expone según su análisis las verdaderas razones del caso de Libia, entendiendo que "[...] sería mucho más pertinente tratar a Libia como un estudio de caso sobre las formas en que las intervenciones supuestamente limitadas tienden a convertirse en campañas para el cambio de régimen" (Zenko, 2016). Cabe aclarar que un factor determinante en la diferencia de los análisis es el tiempo, puesto que las reseñas positivas tuvieron lugar en el mismo año (incluso mes), a diferencia de las críticas que se dieron ya pasados alrededor de 4 y 5 años, en donde las consecuencias son mucho más claras.

\section{Rusia dentro del uso de la responsabilidad de proteger}

En primer lugar, según perspectivas académicas, se evidencia que Rusia ha sido uno de los países con más reservas al momento de tomar la decisión de actuar en nombre de la responsabilidad de proteger. Esto es relevante en la medida en que Rusia hace parte de los miembros permanentes del Consejo de Seguridad, a pesar de que en los estatutos de la responsabilidad de proteger se explicita la recomendación de evadir el veto, ya que "la idea es que los miembros permanentes, cuando sus intereses vitales no se vean comprometidos por un asunto, no harán uso de su derecho de veto para obstaculizar la aprobación de una resolución respaldada por la mayoría" (Comisión Internacional sobre Intervención y Soberanía de los Estados, 2001, p. 56). Se entiende que a pesar de esto, al ser circunstancias que requieren acciones concisas e inmediatas, se contemplan otras posibilidades como que la Asamblea General apoyara la intervención como Unión Pro Paz. Otra acción alternativa podría ser que las organizaciones regionales tomaran cartas en el asunto e intervinieran moderadamente.

Para entender las razones por las cuales Rusia tiende a optar por el veto, es necesario partir del reconocimiento de los intereses que tiene. Según 
aproximaciones académicas previas, se ha llegado a la conclusión de que Rusia, a pesar de brindar apoyo a la implementación de la responsabilidad de proteger por medio de discursos y comunicados, en términos fácticos no ha cumplido con la exigencia requerida, por el número de vetos que ha otorgado. Según los análisis, no se puede dejar de lado la trayectoria y las experiencias previas que ha tenido Rusia en la historia, en donde la política doméstica es supremamente importante y el concepto de soberanía es fundamental y muy poco negociable, ya que "En algunos casos, este argumento enmascara la renuencia de Rusia (y no solo de Rusia) a sobreesfuerzo de recursos internacionales. Más peligrosamente, este énfasis puede ser invocado por Moscú a 'Proteger' un régimen dado de la interferencia externa" (Baranovsky y Mateiko, 2016, p. 52).

Ejemplo de lo anterior fue en el 2008 cuando Myanmar se vio fuertemente afectado por un ciclón y Rusia se opuso a la aplicación de la responsabilidad de proteger en términos de intervención "[...] porque las autoridades del país, por razones de seguridad, se negaron a aceptar la asistencia internacional" (Baranovsky y Mateiko, 2016, p. 53). En el 2006, Rusia se abstuvo de votar por la misión de la ONU en Darfur, debido a que según una declaración oficial del gobierno ruso,

Uno de los principios fundamentales del mantenimiento de la paz de la ONU es obtener el consentimiento de un país receptor, que en este caso es el gobierno legítimo de Sudán, para llevar a cabo las operaciones de mantenimiento de la paz. Jartum, sin embargo, aún no ha dado su consentimiento para una operación de la ONU en Darfur, lo que puede crear dificultades en la etapa de implementación práctica de esta resolución (Embajada de la Federación Rusa, 2006).

En el 2011, Rusia se abstuvo de votar por la intervención humanitaria en Libia bajo los argumentos de cuáles serían los límites de la participación internacional. En este orden de ideas, Rusia ha actuado en pos de prevenir la utilización indiscriminada de la responsabilidad de proteger. Como bien se mencionó previamente, la concepción de soberanía estatal que tiene Rusia ha demarcado su postura a lo largo de la posible utilización y/o necesidad del régimen de responsabilidad de proteger. En su primer comunicado en junio del 2009, Rusia argumenta que "Hacemos hincapié en la responsabilidad de todas las partes, incluidas las fuerzas extranjeras, para garantizar la seguridad de los civiles y el estricto cumplimiento de las normas del derecho internacional humanitario" (Rogachev, 2009), declaración que deja entrever cierto apoyo a la intervención. Para poder entender este caso se debe analizar lo ocurrido entre Rusia y Georgia, puesto que en el 2008 la crisis entre estos dos Estados se encontraba en su mayor apogeo. Para entrar en contexto hay que especificar que, en el territorio de Osetia del Sur, perteneciente a Georgia, hay fuerzas pro-rusas, las cuales en el año 2008 se vieron atentadas por el ejército de Georgia, según demandas de Rusia ante el Consejo de Seguridad.

Otro detonante del conflicto fue la invasión de territorio aéreo por parte de cuatro aviones militares rusos sobre Osetia, lo que llevó al recrudecimiento del ambiente hostil en la frontera, causando altercados militares entre ambos bandos (Nichol, 2009). El presidente ruso Dmitri Medvédev, ante estas acciones mencionó que "no permitiremos que nuestros compatriotas sean asesinados con impunidad. Aquellos que son responsables de eso serán debidamente castigados". Parecía afirmar el control perpetuo de Rusia al afirmar que "históricamente Rusia ha sido, y continuará siendo, un garante de la seguridad de los pueblos del Cáucaso" (Nichol, 2009, p. 5).

Siendo clara evidencia de la situación en la que se encontró un año antes Rusia, se logra entender el porqué de la aceptación que presenta el ministro de relaciones internacionales, Rogachev, al aprobar las acciones de la responsabilidad de proteger mostrando la importancia de emprender una causa justa y necesaria. En julio del 2009, la declaración del representante de Rusia ante las Naciones Unidas se ve distinta en los componentes de la importancia de llevar a cabo la responsabilidad de proteger. Por otra parte, durante todo el discurso hace hincapié en los propios medios que tienen o deben tener los Estados para asegurar la vida de sus ciudadanos.

Creemos que la responsabilidad inicial de proteger a las personas del genocidio, los crímenes de guerra, la depuración étnica y los crímenes de lesa humanidad recae en los Estados. Los Estados deben fortalecer y expandir constantemente sus propios medios para mantener esa responsabilidad (Churkin, 2009).

Al mismo tiempo, manifiesta su opinión frente a cómo debería tomar cartas en el asunto la 
comunidad internacional: "En nuestra opinión, el papel de la comunidad internacional debería, en primera instancia, centrarse en brindar asistencia integral a los Estados para fortalecer su propia capacidad y la diplomacia preventiva" (Churkin, 2009). En la postura e intereses de Rusia, es fundamental el rol que deben cumplir los Estados, puesto que de no ser así se podría caer en grandes efectos colaterales:

Advertimos en contra de tomar medidas precipitadas e impacientes para aplicar esa idea arbitrariamente a países específicos y en contra de interpretarla de manera demasiado amplia. Eso no solo es contraproducente, sino también peligroso en términos de aprovechar los esfuerzos internacionales para promover la pazy la seguridad internacionales (Churkin, 2009).

Si se sigue este último planteamiento, se podría entender que habría cierto nivel de incredulidad en cuanto a las razones de la intervención y los verdaderos intereses que estas ocultan. Ante este último punto, en el noveno debate abierto sobre la protección de civiles en el año 2010, Churkin observó que:

El cumplimiento firme de todas las partes con las normas del derecho internacional humanitario y las decisiones del Consejo de Seguridad, así como el rechazo de los enfoques selectivo y unilateral en la interpretación de las violaciones, pueden promover mejoras en situaciones de conflicto y fortalecer la protección genuina para civiles en conflictos armados (Churkin, 2010).

De esta manera, da a conocer su énfasis en la objetividad y en la claridad que debe haber en cuanto a los escenarios donde es necesaria e inmediata la acción conjunta de todos los Estados para la prevención de ataques de lesa humanidad. Si partimos de que cada caso es específico y es necesario analizarlo con lupa y pinzas, se podría dar cuenta del porqué en ciertos casos la intervención no es la mejor opción, sino que por el contrario, termina siendo la peor de las posibilidades. Lo anterior se dio en el caso previamente analizado de Libia. Tales fueron las consecuencias negativas que Rusia le recalca de manera crítica y comprometedora en el año 2016 en el panel temático de la Asamblea General de las Naciones Unidas: "Del compromiso a la implementación: diez años de la responsabilidad de proteger".
En este escenario es muy clara la posición en contra de lo efectuado por la Asamblea de Seguridad en el 2011 contra Libia. Empieza la intervención discursiva trayendo a colación los hechos, pero en los mismos términos que se estaban manejando, y es a partir del décimo aniversario del primer congreso donde se acordó la responsabilidad de proteger, y es por medio del aniversario de lo sucedido en Libia, "Hace cinco años fue bombardeado. En 2011, muchos etiquetaron con entusiasmo estos bombardeos como el primer caso de la aplicación práctica del concepto R2P. Actualmente, sin embargo, estos eventos se consideran mejor olvidados" (Delegación de la Federación Rusa ante las Naciones Unidas, 2016). Siguiendo el discurso, se menciona la crítica contundente a la situación y se le agrega cierta culpa a la Secretaría de las Naciones Unidas por haber pasado por desapercibida la situación y los efectos colaterales de esta,

El uso de la fuerza con el pretexto de la protección de civiles condujo a la desintegración completa del Estado libio, sumiendo al país en estragos e inestabilidad.[...] Por eso, no hemos visto un solo intento de análisis franco y exhaustivo de lo sucedido en Libia, ni por parte de la Secretaría ni por parte de los Estados miembros (Delegación de la Federación Rusa ante las Naciones Unidas, 2016).

Continuando con el discurso, se menciona la clara oposición por parte de Rusia hacia la aplicación de la responsabilidad de proteger, puesto que de todos los intentos no se ha llegado a un consenso: "El diálogo interactivo anual sobre R2P se ha convertido completamente en el monólogo de los partidarios de este concepto. Digo el monólogo no porque otros puntos de vista no estén presentes, sino porque se oyen, pero no se escuchan" (Delegación de la Federación Rusa ante las Naciones Unidas, 2016). En este caso, es clara la oposición que existe en el escenario de las instituciones internacionales a la responsabilidad de proteger, puesto que la soberanía sigue teniendo un rol importante en las relaciones de poder y cooperación entre los actores del sistema internacional. A pesar de que se ha llegado a consensos, el tema de territorialidad e identidad sigue pesando sobre lo que se considera el bien común. Si se analiza por ambas partes, tanto Estados Unidos como Rusia actúan de acuerdo a sus intereses. 
La relaciones diplomáticas, económicas y de intercambio que influyeron en la posición de Rusia frente al régimen colectivo que actúa en nombre de la responsabilidad de proteger se refieren a que este imprimió parte del dinero de Libia. A pesar del embargo armamentístico por parte de las Naciones Unidas, Libia sigue teniendo libre circulación de armas gracias al apoyo que Rusia le ha brindado al régimen político de Tobruk; de igual forma, las relaciones diplomáticas se mantienen en alianza entre estos dos Estados (Saini, 2016). Estas interacciones son determinantes en el momento de entender por qué los Estados toman decisiones y posturas, a partir de la interacción que se establezca con el otro y de los intereses que comparten. Así mismo, se genera una identidad que también determina si el otro es amigo o enemigo. En el caso de Rusia y Libia, se revelan otros posibles intereses ocultos,

Es poco probable que el Kremlin solo esté interesado en aumentar su mercado de armamentos; más bien, tener un papel central en la reconstrucción de Libia, tanto política como económicamente, es el verdadero premio. Después de años de guerra, Libia está en un lugar difícil y necesita una reconstrucción casi completa, no solo a nivel social y político, sino también en términos de infraestructura (carreteras, ferrocarriles, ciudades). Aunque ha Estado en declive durante años, la explotación de petróleo $\mathrm{y}$ gas en Libia tiene un enorme potencial. Sin mencionar que un gobierno dispuesto podría garantizar el uso de los puertos libios orientales de Sirte y Benghazi en el Mediterráneo. Para Rusia, que continúa expandiendo su presencia en el Mediterráneo oriental (específicamente en Siria), estas oportunidades son una potencial mina de oro estratégica (Saini, 2016).

Se entiende en primer lugar que son interacciones sociales y para analizarlas es importante tener en cuenta que "hace sentido incluir en el concepto de discurso no sólo elementos observables verbales y no verbales, o interacciones sociales y actos de habla, sino también las representaciones cognitivas y estrategias involucradas durante la producción o comprensión del discurso" (Meersohn, 2005, p. 291). La teoría del constructivismo funciona para entender cómo es la concepción de ciertos términos como lo es la seguridad y el deber ser, por medio de la utilización del análisis del discurso para esclarecer la forma como se entienden y construyen los ideales y conceptos dentro de la esfera social y diplomática.
Este último ha sido evocado principalmente por las Naciones Unidas en cuanto a intervención humanitaria se refiere. La necesidad de crear este régimen internacional se da por la necesidad de inmediata o pronta respuesta a ataques que impliquen ser genocidios, llegando al punto de evitarlos. Según la Comisión Internacional sobre Intervención y Soberanía de los Estados, en el año 2001 se brindaron los principios esenciales, los criterios de acción y operacionales del régimen. $\mathrm{Y}$ es allí donde surge la necesidad del concepto de responsabilidad de proteger, siendo:

Importante por tres motivos: en primer lugar, implica que las autoridades estatales son responsables de proteger la seguridad y la vida de los ciudadanos y promover su bienestar; en segundo lugar, sugiere que las autoridades políticas nacionales son responsables ante los ciudadanos a nivel interno y ante la comunidad internacional a través de las Naciones Unidas; y, en tercer lugar, significa que los agentes del Estado son responsables de sus actos, es decir, que han de rendir cuentas de sus actos u omisiones (Comisión Internacional sobre Intervención y Soberanía de los Estados, 2001).

En el uso de este régimen se evidencia una dicotomía en cuanto a la aceptación en un primer momento, pero cuando se entiende como intervención, llega a generar controversia por las diversas formas en que se entiende y aplica. La importancia de lo anterior radica claramente en el nivel de entendimiento y uso de dichos términos, puesto que variedad de políticas públicas e internacionales han sido creadas para responder a la defensa territorial, humanitaria y social, llevando así a que tanto los Estados como los individuos deban tomar una postura política o ideológica basada en sus intereses, expectativas e identidades.

\section{Conclusión}

Los Estados se fundamentan en el bienestar común. Si este se ve alterado por cualquiera que sea la circunstancia, debe ser combatido con estrategias que le permitan el mejoramiento, es por ello que se crean políticas, pero el error está cuando estas políticas son individuales y buscan un bien particular y no un bien comunal, como lo harían las políticas colectivas. De esta manera se genera desigualdad entre países, beneficiando a los más ricos y dejando de lado al gran porcentaje de personas humildes. La idea es manejar el Estado 
de manera gradual, hasta lograr que los Estados actúen con políticas que traigan beneficios a la comunidad.

Esta comparación en la percepción que se tuvo del caso de Libia, es para recalcar que, dentro de las interacciones en el sistema internacional, las percepciones juegan un rol importante y la forma en que se construyen da luces de la construcción y mutación de posturas. Claro está que la identidad de Estados Unidos y sus intereses han mostrado cambios con la nueva presidencia de Donald Trump, puesto que en la medida en que las interacciones y el lenguaje entre los actores cambien, así mismo cambiarán también las percepciones tanto a nivel doméstico como internacional.

De los anteriores planteamientos y análisis se deduce que, como lo afirma Wendt, la anarquía es una cualidad del sistema internacional, ya que al no haber poderes supraestatales que obliguen a los Estados a actuar de una manera $\mathrm{u}$ otra, estos son aquellos que se regulan unos a otros. Esto es claramente demostrable en la constitución de regimenes colectivos donde se establecen una serie de normas y principios que representen los intereses de todos los involucrados, pero a pesar de estos intentos el ambiente global tiende ser hostil, ya que a pesar de no llegar a un consenso se efectúan acciones que al final no son representativas a las opiniones de los actores. En palabras de Wendt,

El grado de compromiso con cada una de las identidades particulares y la relevancia de cada una de ellas varía, pero cada identidad es una definición inherentemente social del actor basada en las teorías que los actores mantienen colectivamente sobre ellos mismos, y cada uno sobre los demás que constituyen la estructura del mundo social (Wendt, 1992, p. 8).

Es a partir de este tipo de análisis que surge la relevancia de lo que se calla, de lo que se oculta, tal y como son los intereses de los Estados Unidos y Rusia en imponer perspectivas desde el uso del lenguaje del conflicto y las problematicas diplomáticas, o defender ciertas posturas desde el ámbito diplomático, pero partiendo de unos objetivos económicos o militares, que sin lugar a dudas ha alterado el orden conflictivo de los países y se ha llevado consigo la violación de los derechos humanos de los ciudadanos y fuerza armada involucrada.

Para tener la posibilidad de ver tras bambalinas en el gran escenario internacional entre
Estados Unidos y Rusia, se deben tener presentes todas las relaciones constitutivas y el entramado que sostienen las relaciones entre los actores. De igual manera, el análisis del discurso de Obama, permite revelar cómo son las percepciones en escena, quién es el enemigo, quién es la amenaza, quién necesita a quién y quién domina a quién; es por medio del discurso que uno entiende que hay aspectos que son endógenos a los Estados, es decir, que las percepciones son construidas por ellos mismos y no son modelos impuestos o intrínsecos al sistema internacional, sino que por el contrario, se construyen el uno al otro.

\section{Referencias}

Amstutz, M. R. (2013). Concepts, Theories, and Cases in Global Politics. International Ethics. Maryland: Rowman \& Littlefield Publishers, Inc.

Baranovsky, V., \& Mateiko, A. (2016). Responsibility to Protect: Russia's Approaches. The International Spectator, 49-69.

BBC. (2011, marzo 20). Libya: US, UK and France attack Gaddafi forces. Retrieved from BBC: https:// www.bbc.com/news/world-africa-12796972

Campbell, D. (1992). Writing Security: United States Foreign Policy and the Politics of Identity. Manchester: Manchester University Press.

Churkin, V. (2009). Russian - Statement at the July 2009 GA Debate on RtoP (As Delivered). Retrieved from http://www.responsibilitytoprotect.org/index. $\mathrm{php/component/content/article/133-europe/5460-}$ russias-statements-on-rtop-from-2009-present

Churkin, V. (2010). Statement of the Russian Federation: Ninth Open Debate on the Protection of Civilians in Armed Conflict. Retrieved from http://www.responsibilitytoprotect.org/Russian $\% 20$ Federation \%20-\%20Ninth\%200pen \%20 Debate\%20on\%20the\%20Protection\%20of\%20 Civilains\%20in\%20Armed\%20Conflict.pdf

Comisión Internacional sobre Intervención y Soberanía de los Estados. (2001). La responsabilidad de proteger

Cooper, H., y Myers, S. L. (2011). U.S. Tactics in Libya May Be a Model for Other Efforts. Retrieved from https://www.nytimes.com/2011/08/29/world/ africa/29diplo.html

Delegación de la Federación Rusa ante las Naciones Unidas. (2016, febrero 26). Statement delivered by the Delegation of the Russian Federation to the United Nations, at the United Nations General Assembly Thematic Panel Discussion. Retrieved from http://responsibilitytoprotect.org/ $\underline{\text { Russian\%20Statement(1).pdf }}$ 
Ellison, K. (2011, agosto 26). U.S. role made the difference in Libya. Retrieved from http://edition.cnn. com/2011/OPINION/08/26/ellison.libya.obama/ index.html

Embajada de la Federación Rusa. (2006, enero 11). Russia's position on Darfur. Retrieved from https://www.rusemb.org.uk/press/40

Graebner, N. A., Dean Burns, R. y Siracusa, J. (2008). Reagan, Bush, Gorbachev: Revisiting the end of the cold war (Greenwood, 2008).

Hansen, L. (2007). Security as Practice. Discourse Analysis and the Bosnian War. New York: The Cromwell Press.

Hernández, E. (2006). "Reconstrucción de una política exterior de Estado en México: la vía nacional". Relaciones Internacionales, núm. 96.

Judt, T. (2010). Algo va mal. https://marcelagonzalezduarte. files.wordpress.com/2018/01/algo-va-mal-tonyjudt.pdf

Korab-Karpowicz, W. J. (2017, mayo 24). Political Realism in International Relations. Retrieved from https://plato.stanford.edu/entries/realism-intlrelations/

Kuperman, A. J. (2015, marzo-abril). Obama's Libya Debacle, How a Well-Meaning Intervention Ended in Failure. Retrieved from https://www. foreignaffairs.com/articles/libya/obamas-libyadebacle

López-Jacoiste, E. (2011). La crisis de Libia desde la perspectiva de la responsabilidad de proteger. Anuario español del derecho internacional, 109-152.

Luck, E. C. (2009). Sovereignty, Choice, and the Responsibility to Protect. Global Responsibility to Protect I, 10-21. Koninklijke Brill NV, Leiden. https://www.ipinst.org/images/pdfs/s3.pdf

McSweeney, B. (1999). Security, Identity and Interests. New York: Cambridge University Press.

Meersohn, C. (2005). Introducción a Teun Van Dijk: Análisis de Discurso. Moebio, 288-302.

Nichol, J. (2009). Russia-Georgia Conflict in August 2008: Context and Implications for U.S Interests. Library Congress. Congressional Research Service. Public Domain.

https://www.hsdl.org/?view\&did=37806

Obama, B. (2011, marzo 28). Remarks by the President in Address to the Nation on Libya. Retrieved from https://obamawhitehouse.archives.gov/photosand-video/video/2011/03/28/president-obama-sspeech-libya\#transcript

Pérez, R. C. (2005). Elementos básicos para un constructivismo social. Avances en Psicología Latinoamericana, 43-61.

Peterson, J. W. (2017). Russian-American relations in the post-Cold War world. Manchester University Press.

Rogachev, I. (2009, junio). Russia's First Statement at
June 2009 Security Council Open Debate on Protection of Civilians. Retrieved from http://www.responsibilitytoprotect.org/index.php/ component/content/article/133-europe/5460russias-statements-on-rtop-from-2009-present

Romero, Y. H., y Sosa, R. V. (2007). El concepto de intersubjetividad en Alfred Schutz. Espacios Públicos, 228-240.

Saini, F. (2016, septiembre 1). Russia and Libya: A brief history of an on-again-off-again friendship. Retrieved from https://www.brookings.edu/blog/ order-from-chaos/2016/09/01/russia-and-libya-abrief-history-of-an-on-again-off-again-friendship/

United Nations Office on Genocide Prevention and the Responsability to Protect. (n.d.). Responsability to Protect. Retrieved from http://www.un.org/ en/genocideprevention/about-responsibility-toprotect.html

Waltz, K. (1979). Theory of International Politics. University of California, Berkeley. Addison-Wesley.

Wendt, A. (1992). Anarchy is what states made of it: the social construction of power politics. International Organization, 391-425.

Wendt, A. (2005). La anarquía es lo que los estados hacen de ella. La construcción social de la política de poder. Revista Académica de Relaciones Internacionales, núm. 1.

https://revistas.uam.es/index.php/ relacionesinternacionales/article/ download $/ 4828 / 5297$

Zenko, M. (2016, marzo 22). The Big Lie About the Libyan War. Retrieved from https://foreignpolicy. com/2016/03/22/libya-and-the-myth-ofhumanitarian-intervention/

Zoubir, Y. H., y Murillo, L. (2012). El colapso de la dictadura de Gadafi ¿Qué futuro para Libia? Foro Internacional, 361-378. 